\title{
Costs of chronic obstructive pulmonary disease in urban areas of China: a cross-sectional study in four cities
}

This article was published in the following Dove Press journal:

International Journal of COPD

19 October 2016

Number of times this article has been viewed

\author{
Xiaoying Chen ${ }^{1,2}$ \\ Na Wang ${ }^{1,2}$ \\ Yue Chen ${ }^{3}$ \\ Tian Xiao ${ }^{1,2}$ \\ Chaowei $\mathrm{Fu}^{1,2}$ \\ Biao $\mathrm{Xu}^{1,2}$ \\ 'Department of Epidemiology, School \\ of Public Health, ${ }^{2}$ Key Laboratory of \\ Public Health Safety, Fudan University, \\ Shanghai, People's Republic of China; \\ ${ }^{3}$ Department of Epidemiology, Faculty \\ of Medicine, University of Ottawa, \\ Ottawa, ON, Canada
}

Correspondence: Chaowei Fu Department of Epidemiology, School of Public Health, Fudan University, Box 289, 138 Yi Xue Yuan Road, Shanghai 200032, People's Republic of China

Tel/fax +86 2I 5423 78I I

Email fcw@fudan.edu.cn
Purpose: The economic burden of COPD has not been well studied in China. This study investigated the total costs caused by COPD and the influencing factors for the high economic burden in urban areas of China.

Patients and methods: A cross-sectional study was carried out among 678 COPD patients in four cities in China in 2011. The average annual direct medical costs (DMCs), direct nonmedical costs (DNMCs), and indirect costs (ICs) on COPD were measured by median and mean ( \pm standard deviation). Logistic regression model was used to explore factors related to high total costs on COPD.

Results: The median annual DMCs, DNMCs, and ICs per COPD patient were RMB 5565 Yuan (US\$ 862), 0 Yuan (US\$ 0), and 0 Yuan (US\$ 0), respectively, and the mean annual DMCs, DNMCs, and ICs per COPD patient were RMB $11968( \pm 22422)$ Yuan [US\$ $1853( \pm 3472)], 539( \pm 2092)$ Yuan [US\$ $83( \pm 324)$ ], and 2087 ( \pm 8110$)$ Yuan [US\$ $323( \pm 1256)$ ], respectively. The annual DMCs, DNMCs, and ICs for diagnosed COPD patients were RMB 195.70 billion Yuan (US\$ 30.30 billion), 8.78 billion Yuan (US\$ 1.36 billion), and 34.10 billion Yuan (US\$ 5.28 billion), respectively, in China. Hospitalization accounted for $56.7 \%$ of the total costs. High economic burden was significantly related to age, acute exacerbations, and disease severity in COPD patients.

Conclusion: COPD posed a heavy economic burden in China. Measures to delay the disease progression and to reduce the risks of acute exacerbation and hospitalization will help substantially lower the costs for COPD care.

Keywords: economic burden, hospitalization, acute exacerbations, disease severity

\section{Introduction}

COPD is characterized by a progressive deterioration of lung function with some mental and physical comorbidities such as depression, dystrophy, and heart failure. ${ }^{1}$ COPD is one of the important public health problems because of the high prevalence, mortality, and economic burden. The World Health Organization estimated that $>3$ million people worldwide died of COPD in 2012, equal to $6 \%$ of all deaths globally that year, and $>90 \%$ of COPD deaths occurred in low- and middle-income countries, including China. ${ }^{2}$ In China, a nationwide survey showed that the prevalence of COPD among people over 40 years old was $8.2 \%$ (12.4\% for men and $5.1 \%$ for women) in $2008 .^{3}$ Based on the results of the Global Burden of Diseases Study, COPD was the third leading cause of death in China in $2010 .^{4}$

COPD is a disease associated with substantial economic burden worldwide. ${ }^{5,6}$ Previous studies conducted in China showed that COPD was also costly and imposed an enormous economic burden on both families and society in the country. The average 
of annual direct medical costs (DMCs) and direct nonmedical costs (DNMCs; including costs caused by nutrition, transportation, and caregivers) in 2006 were RMB 11744 Yuan (US\$ 1473) and 1570 Yuan (US\$ 197), respectively, for each COPD patient living in urban areas of China. The average work loss was 17 days for COPD patients and 14 days for their relatives in 2006. ${ }^{7}$ Previous studies had indicated that hospitalization is a major driver of DMCs for COPD and such DMCs are significantly correlated with the comorbidity and severity of COPD. ${ }^{8-11}$ However, there have been so far limited data on the costs due to COPD in China, including direct and indirect costs (ICs) and factors related to the costs. This cross-sectional study was designed and conducted to provide the information for urban communities of China.

\section{Patients and methods}

\section{Study population}

This multi-center cross-sectional study was carried out in four megacities, ie, Beijing, Guangzhou, Shanghai, and Chengdu, of China in 2011. Fifteen community health centers or hospitals participated in recruiting subjects from local communities. A total of $721 \mathrm{COPD}$ patients were invited and $94 \%$ of them were eligible based on the inclusion criteria (local residents who lived in the city for at least 2 years without any unstable comorbidities or any severe conditions needed to be treated as inpatients). COPD cases who did not meet the inclusion criteria were excluded. ${ }^{12}$ A written informed consent was obtained from all subjects. They were free to withdraw from the study at any time with no negative consequences. The ethics approval for this study was issued by the Institutional Review Board of the Fudan University School of Public Health. Further details are available in a previous publication. ${ }^{12}$

\section{Data collection}

Health workers were recruited as data collectors from each study site after they were trained by academic investigators. A structured questionnaire was used to collect direct medical and nonmedical costs as well as ICs. Investigators interviewed subjects, reviewed medical charts, and obtained detailed information on medical care utilization, transportation/nutrition costs, and work loss of COPD patients and caregivers. Free lung function testing was provided to all subjects during the survey.

\section{Cost definition and estimation}

The total costs of COPD included direct costs (DMCs and DNMCs) and ICs. In this study, the annual DMCs were all medical expenditures incurred by outpatient clinical visit, emergency, and hospitalization due to COPD, namely the total expenditures for treating COPD, including copayments, diagnoses, treatments, diagnostic tests, prescription drugs, and medical supplies in the previous 12 months. The annual DNMCs were all nonmedical expenditures including costs for transportation and costs for living care and nutrition. ICs referred to the costs caused by lost productivity, including work loss of patients and relatives as their caregivers, for which the authors used average day wages in 2010 (Beijing: RMB 65168/250 Yuan [US\$ 10090/250]; Shanghai: 66115/250 Yuan [US\$ 10236/250]; Chengdu: 32567/250 Yuan [US\$ 5042/250]; and Guangzhou: 40432/250 Yuan [US\$ 6260/250]) to estimate the ICs. ${ }^{13}$ The cost figures were expressed in RMB and equivalent US dollars based on the average exchange rate of the study year.

\section{Statistical analysis}

All data were double input into a database in EpiData3.1 Chinese version. Statistical analysis was completed using SPSS22. For continuous outcomes, median and/or mean \pm standard deviation were used to describe the data, and Kruskal-Wallis or Wilcoxon test was used to test the differences of costs among groups. For categorical outcomes, frequencies (percentages) were provided and chi-square test was used to test different distributions. The upper quartile of total costs for COPD was calculated, and costs not less than top 25 th percentile were defined as being high economic burden. Logistic regression model was used to calculate the crude odds ratio, adjusted odds ratio (aOR), and 95\% confidence intervals (CIs) for factors associated with high economic burden. $P<0.05$ was used to define statistical significance.

\section{Results}

\section{Characteristics of COPD patients}

Of the 678 participants, about a quarter of them were from four cities (Beijing: 25.1\%; Guangzhou: 25.8\%; Shanghai: 24.8\%; and Chengdu: $24.3 \%$ ). A majority of them were male $(72.9 \%)$ and $>60$ years old $(82.9 \%)$. Approximately threefifths of them had received $\leq 9$ years of education $(61.6 \%)$ and had monthly household income per capita between RMB 1,000 Yuan (US\$ 154) and 2,999 Yuan (US\$ 464) (60.7\%). One-fifth (19.4\%) remained current smokers. Almost all the patients $(95.6 \%)$ had at least one type of medical insurances. The median duration of COPD was 4.0 years. A majority of the COPD patients had at least one comorbid chronic disease (79\%). According to the criteria of the Global Initiative for Chronic Obstructive Lung Disease, $20.6 \%$ of them were in mild stage, $47.3 \%$ of them were in moderate stage, $26.7 \%$ of them were in severe stage, and $9.0 \%$ of them were in very severe stage (Table 1). 
Table I Demographic and clinical characteristics of COPD patients

\begin{tabular}{|c|c|c|c|}
\hline & Male, n (\%) & Female, n (\%) & $\overline{\text { Total, n (\%) }}$ \\
\hline \multicolumn{4}{|l|}{$\overline{C i t y}$} \\
\hline Beijing & $100(20.2)$ & $70(38.0)$ & $170(25.1)$ \\
\hline Guangzhou & $152(30.8)$ & $23(12.5)$ & $175(25.8)$ \\
\hline Shanghai & $125(25.3)$ & $43(23.4)$ & $168(24.8)$ \\
\hline Chengdu & $117(23.7)$ & $48(26.1)$ & $165(24.3)$ \\
\hline Age ( $>60$ years $)$ & $417(85.3)$ & $140(76.5)$ & $557(82.9)$ \\
\hline \multicolumn{4}{|l|}{ Education (years) } \\
\hline$\leq 9$ & $290(58.7)$ & $127(69.4)$ & $417(61.6)$ \\
\hline $10-12$ & $122(24.7)$ & $42(23.0)$ & $164(24.2)$ \\
\hline$>12$ & $82(16.6)$ & $14(7.7)$ & $96(14.2)$ \\
\hline \multicolumn{4}{|c|}{ Monthly household income per capita, RMB Yuan (US\$) } \\
\hline$<1,000(154)$ & $30(6.1)$ & $9(4.9)$ & $39(5.8)$ \\
\hline $1,000-1,999(154-308)$ & $132(26.9)$ & $65(35.5)$ & $197(29.3)$ \\
\hline $2,000-2,999(309-463)$ & $152(31.0)$ & $59(32.2)$ & $2 I I(3 I .4)$ \\
\hline $3,000-3,999(464-618)$ & $74(15.1)$ & $20(10.9)$ & $94(14.0)$ \\
\hline$\geq 4,000(6 / 9)$ & $102(20.8)$ & $30(16.4)$ & $132(19.6)$ \\
\hline Retired & $418(85.1)$ & $155(85.6)$ & $573(85.3)$ \\
\hline Married & $435(88.2)$ & $137(74.5)$ & $572(84.5)$ \\
\hline Current smoker & $119(24.1)$ & $12(6.5)$ & 131 (19.4) \\
\hline Regular exercise & $232(47.5)$ & $61(33.5)$ & $293(43.7)$ \\
\hline Having medical insurance & 47I (95.3) & $177(96.2)$ & $648(95.6)$ \\
\hline $\begin{array}{l}\text { Course of COPD } \\
\text { (years)* }\end{array}$ & $4.0(8.9 \pm 12.0)$ & $4.0(9.1 \pm 10.7)$ & $4.0(8.9 \pm$ I I.6) \\
\hline \multicolumn{4}{|l|}{ Comorbidities } \\
\hline 0 & $123(24.9)$ & $19(10.4)$ & $\mid 42(2 \mid .0)$ \\
\hline I & $172(34.8)$ & $61(33.3)$ & $233(34.4)$ \\
\hline 2 & $124(25.1)$ & $46(25.1)$ & $170(25.1)$ \\
\hline$\geq 3$ & $75(15.2)$ & $57(31.1)$ & $132(19.5)$ \\
\hline \multicolumn{4}{|l|}{ Severity of COPD } \\
\hline Mild & $88(\mid 7.8)$ & $52(28.3)$ & I 40 (20.6) \\
\hline Moderate & $215(43.5)$ & $81(44.0)$ & $296(43.7)$ \\
\hline Severe & $139(28.1)$ & $42(22.8)$ & $181(26.7)$ \\
\hline Very severe & $52(10.5)$ & $9(4.9)$ & $61(9.0)$ \\
\hline Total & $494(72.9)$ & $184(27.1)$ & $678(100.0)$ \\
\hline
\end{tabular}

Note: *Data were presented as median (mean \pm standard deviation). Abbreviation: COPD, chronic obstructive pulmonary disease.

\section{Costs for COPD}

Of the 678 subjects, $68.5 \%$ visited a department of outpatient at least once during the past 3 months and $40.9 \%$ had hospitalization during the past year prior to the survey. The median annual direct costs, ICs, and total costs per patient due to COPD were RMB 6,096 Yuan (US\$ 944), 0 Yuan (US\$ 0), and 6,405 Yuan (US\$ 992), respectively (Table 2). Direct costs accounted for $86 \%$ of the total costs, and hospitalization costs were a major driver of the direct costs, accounting for $65.9 \%$ (Figure 1). During the past 12 months, $12.8 \%$ of the patients reported that either they or their relatives were off work with a total of 5,944 workdays or a median of 2.2 workdays per patient because of their COPD. Caregivers' work loss accounted for $60.7 \%$ (Table 2).

\section{COPD economic burden in China}

The ratio of annual total costs for COPD versus annual average income was similar for Beijing (34.1\%), Guangzhou (32.8\%), and Chengdu (32.3\%) and was much lower for Shanghai (19.2\%). There were 568 million people aged $\geq 40$ years in China in 2010 , and it was estimated that $8.2 \%$ of them or 46.6 million people had COPD, of whom only $35.1 \%$ or 16.3 million COPD had been diagnosed. ${ }^{3}$ For this population of diagnosed COPD, the DMCs, DNMCs, and ICs were estimated to be approximately RMB 195.70 billion Yuan (US\$ 30.30 billion), 8.78 billion Yuan (US\$ 1.36 billion), and 34.10 billion Yuan (US\$ 5.28 billion), respectively. There may be a big difference in COPD care costs between large cities and other areas of the country.

\section{COPD costs associated with patients' characteristics}

Costs for outpatient visit and hospitalization were significantly correlated with age, household income, severity of COPD, and comorbidity. The costs increased with the number of comorbidities (annual DMCs, DNMCs, ICs, and total costs) and severity (annual DMCs, DNMCs, and total costs). Costs per outpatient visit were higher and costs per hospitalization were lower for patients aged $>60$ years. The annual DNMCs and ICs were higher in women than in men but the annual DMCs showed no sex difference. The annual DMCs and total costs were higher in patients aged $>60$ years (Table 3 ).

Logistic regression model was further applied to explore possible influencing factors for high total costs due to COPD (Table 4). After adjustment for study site and other covariates, there was a significantly greater risk of high costs for older patients $(>60$ years) than for young patients $(\mathrm{aOR}=2.36$, $P=0.015)$. The risk of high costs increased significantly with the severity of COPD and the number of exacerbations. The aOR for high costs was 4.68 (95\% CI: 2.71-8.08) for COPD patients with an acute exacerbation versus those without an acute exacerbation.

\section{Discussion}

COPD is a well-known disease of high economic burden worldwide. This study demonstrated that COPD posed a huge economic burden in China, including RMB 195.70 billion Yuan (US\$ 30.30 billion) of DMCs, 8.78 billion Yuan (US\$ 1.36 billion) of DNMCs, and 34.10 billion Yuan (US\$ 5.28 billion) of ICs in 2010. In China, the total health expenditure (THE) was RMB 1998.04 billion Yuan (US\$ 309.35 billion) in 2010, accounting for $4.98 \%$ of GDP, and the THE per capita was RMB 2315.5 Yuan (US\$ 358.50) and 666.3 Yuan (US\$ 103.16) in urban and rural areas, respectively. ${ }^{14}$ Thus, the estimated DMCs of COPD accounted for $9.8 \%$ of THE. In this study, the annual direct expenditure and total costs per patient were equivalent to $26.1 \%$ and $29.7 \%$ of their personal income, respectively, 
Table 2 Annual direct and indirect costs per patient for COPD, RMB Yuan (US\$)

\begin{tabular}{|c|c|c|c|c|c|}
\hline & Beijing & Guangzhou & Shanghai & Chengdu & Total \\
\hline \multirow[t]{2}{*}{ Direct costs } & $7,870(16,462 \pm 28,746)$ & $3,773(12,739 \pm 28,068)$ & $6,288(11,254 \pm 15,355)$ & $7,07 \mid(9,897 \pm \mid 4,462)$ & $6,096(12,614 \pm 22,892)$ \\
\hline & $(I, 2 \mid 8[2,549 \pm 4,45 I])$ & $(584[1,972 \pm 4,346])$ & $(974[1,742 \pm 2,377])$ & $(1,095[1,532 \pm 2,239])$ & $(944[1,953 \pm 3,544])$ \\
\hline \multirow{2}{*}{$\begin{array}{l}\text { Direct medical } \\
\text { costs }\end{array}$} & $6,824(15,519 \pm 28,396)$ & $3, I 78(\mid 2,45 I \pm 27,50 I)$ & $5,214(10,235 \pm 14,628)$ & $6,628(9,57| \pm| 4,376)$ & $5,565(11,968 \pm 22,422)$ \\
\hline & $(1,057[2,403 \pm 4,396])$ & $(492[1,928 \pm 4,258])$ & $(807[1,585 \pm 2,265])$ & $(1,026[1,482 \pm 2,226])$ & $(862[1,853 \pm 3,472])$ \\
\hline \multirow[t]{2}{*}{ Outpatient costs } & $2,939(6,480 \pm 15,748)$ & $1,328(2,856 \pm 3,630)$ & $2,598(4,670 \pm 8,339)$ & $0(854 \pm 3,456)$ & $\mathrm{I}, 278(3,730 \pm 9,48 \mathrm{I})$ \\
\hline & $(455[1,003 \pm 2,438])$ & $(206[442 \pm 562])$ & $(402[723 \pm I, 29 \mid])$ & $(0[132 \pm 535])$ & $(198[578 \pm I, 468])$ \\
\hline \multirow{2}{*}{$\begin{array}{l}\text { Outpatient costs } \\
\text { per visit }\end{array}$} & $434(598 \pm 90 I)$ & $190(278 \pm 377)$ & $212(298 \pm 300)$ & $0(97 \pm 343)$ & $156(319 \pm 567)$ \\
\hline & $(67[93 \pm 139])$ & $(29[43 \pm 58])$ & $(33[46 \pm 46])$ & $(0[15 \pm 53])$ & $(24[49 \pm 88])$ \\
\hline \multirow{2}{*}{$\begin{array}{l}\text { Hospitalization } \\
\text { costs }\end{array}$} & $0(9,039 \pm 24,197)$ & $0(9,649 \pm 27,611)$ & $0(5,565 \pm 12,429)$ & $6,397(8,7|7 \pm| 3,803)$ & $0(8,255 \pm 20,967)$ \\
\hline & $(0[1,399 \pm 3,746])$ & $(0[1,494 \pm 4,275])$ & $(0[862 \pm I, 924])$ & $(990[1,350 \pm 2,137])$ & $(0[1,278 \pm 3,246])$ \\
\hline \multirow{2}{*}{$\begin{array}{l}\text { Hospitalization } \\
\text { costs per visit }\end{array}$} & $0(6,120 \pm 17,754)$ & $0(3,938 \pm 8,317)$ & $0(3,|7| \pm 5,644)$ & $3,695(5,00 I \pm 7,824)$ & $0(4,554 \pm 10,972)$ \\
\hline & $(0[948 \pm 2,749])$ & $(0[6 \mid 0 \pm 1,288])$ & $(0[49 I \pm 874])$ & $(572[774 \pm I, 2 \mid I])$ & $(0[705 \pm 1,699])$ \\
\hline \multirow{2}{*}{$\begin{array}{l}\text { Direct } \\
\text { nonmedical costs }\end{array}$} & $30(680 \pm I, 378)$ & $0(308 \pm 106 \mid)$ & $0(848 \pm 3,69 \mid)$ & $0(334 \pm I, 0 I I)$ & $0(539 \pm 2,092)$ \\
\hline & $(5[105 \pm 2 \mid 3])$ & $(0[48 \pm 164])$ & $(0[13| \pm 57|])$ & $(0[52 \pm 157])$ & $(0[83 \pm 324])$ \\
\hline \multirow[t]{2}{*}{ Nutrition costs } & $0(279 \pm 56 I)$ & $0(233 \pm 78 I)$ & $0(719 \pm 3,687)$ & $0(\mid 42 \pm 54 I)$ & $0(34| \pm I, 9| 4)$ \\
\hline & $(0[43 \pm 87])$ & $(0[36 \pm|2|])$ & $(0[1 I I \pm 57 \mid])$ & $(0[22 \pm 84])$ & $(0[53 \pm 296])$ \\
\hline \multirow[t]{2}{*}{ Caregiver costs } & $0(335 \pm 984)$ & $0(59 \pm 458)$ & $0(85 \pm 306)$ & $0(188 \pm 856)$ & $0(166 \pm 718)$ \\
\hline & $(0[52 \pm 152])$ & $(0[9 \pm 7 I])$ & $(0[13 \pm 47])$ & $(0[29 \pm 133])$ & $(0[26 \pm I I I])$ \\
\hline \multirow{2}{*}{$\begin{array}{l}\text { Transportation } \\
\text { costs }\end{array}$} & I $(67 \pm|| 4)$ & $0(16 \pm 140)$ & $0(44 \pm 85)$ & $0(5 \pm 22)$ & $0(33 \pm 104)$ \\
\hline & $(0[10 \pm 18])$ & $(0[2 \pm 22])$ & $(0[7 \pm \mid 3])$ & $(0[I \pm 3])$ & $(0[5 \pm \mid 6])$ \\
\hline \multirow[t]{2}{*}{ Indirect costs } & $0(5,737 \pm 12,532)$ & $0(558 \pm 3,135)$ & $0(1,456 \pm 8,436)$ & $0(6 \mid 4 \pm 3,058)$ & $0(2,087 \pm 8, I \mid 0)$ \\
\hline & $(0[888 \pm 1,940])$ & $(0[86 \pm 485])$ & $(0[225 \pm I, 306])$ & $(0[95 \pm 473])$ & $(0[323 \pm I, 256])$ \\
\hline \multirow[t]{2}{*}{ Due to patient } & $0(2,191 \pm 6,228)$ & $0(52 \pm 557)$ & $0(882 \pm 7,958)$ & $0(275 \pm 2,647)$ & $0(843 \pm 5,240)$ \\
\hline & $(0[339 \pm 964])$ & $(0[8 \pm 86])$ & $(0[137 \pm 1,232])$ & $(0[43 \pm 4 \mid 0])$ & $(0[|3| \pm 8||])$ \\
\hline \multirow[t]{2}{*}{ Work loss, days } & $0(8.4 \pm 6.0)$ & $0(0.3 \pm 0.9)$ & $0(3.3 \pm 7.5)$ & $0(2.1 \pm 5.1)$ & $0(3.5 \pm 5.4)$ \\
\hline & $(0[8.4 \pm 6.0])$ & $(0[0.3 \pm 0.9])$ & $(0[3.3 \pm 7.5])$ & $(0[2.1 \pm 5.1])$ & $(0[3.5 \pm 5.4])$ \\
\hline \multirow[t]{2}{*}{ Due to family } & $0(3,567 \pm 9,739)$ & $0(506 \pm 2,998)$ & $0(578 \pm 2,984)$ & $0(339 \pm I, 59 \mid)$ & $0(1,247 \pm 5,522)$ \\
\hline & $(0[552 \pm I, 508])$ & $(0[78 \pm 464])$ & $(0[89 \pm 462])$ & $(0[52 \pm 246])$ & $(0[193 \pm 855])$ \\
\hline \multirow[t]{2}{*}{ Work loss, days } & $0(13.7 \pm 9.3)$ & $0(3.1 \pm 4.6)$ & $0(2.2 \pm 2.8)$ & $0(2.6 \pm 3.1)$ & $0(5.4 \pm 5.7)$ \\
\hline & $(0[13.7 \pm 9.3])$ & $(0[3.1 \pm 4.6])$ & $(0[2.2 \pm 2.8])$ & $(0[2.6 \pm 3.1])$ & $(0[5.4 \pm 5.7])$ \\
\hline \multirow[t]{2}{*}{ Total } & II,038 $(22,199 \pm 35,174)$ & $3,773(13,277 \pm 28,759)$ & $6,473(12,676 \pm 17,623)$ & $7,200(10,510 \pm 14,934)$ & $6,405(14,695 \pm 25,942)$ \\
\hline & $(1,709[3,437 \pm 5,446])$ & $(584[2,056 \pm 4,453])$ & $(I, 002[1,963 \pm 2,729])$ & $(I, I \mid 5[|, 627 \pm 2,3| 2])$ & $(992[2,275 \pm 4,0 \mid 7])$ \\
\hline
\end{tabular}

Notes: Data were presented as median (mean \pm standard deviation). Kruskal-Wallis or Wilcoxon test was used to test the differences of costs among groups. Abbreviation: COPD, chronic obstructive pulmonary disease.

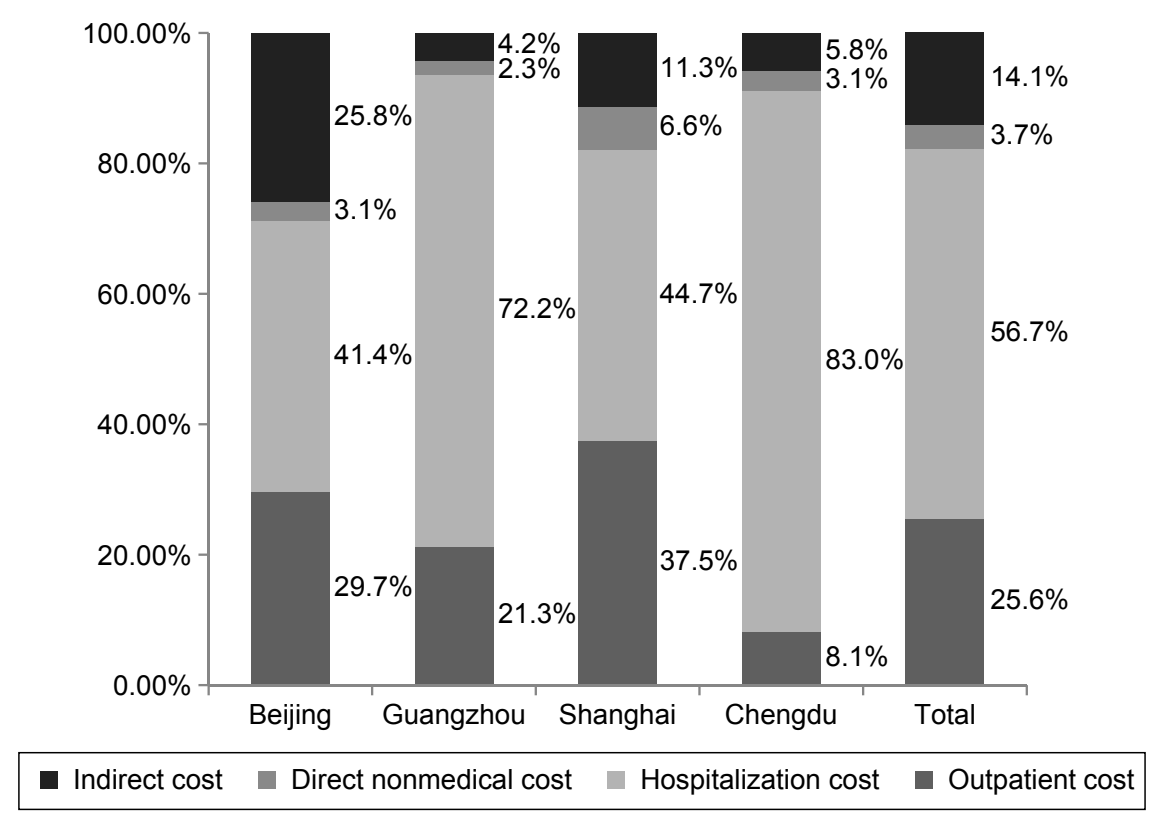

Figure I The proportion of the component of chronic obstructive pulmonary disease-related costs. Note: The costs were the summary of 678 patients. 
Table 3 Costs of COPD by demographic category, RMB Yuan (US\$)

\begin{tabular}{|c|c|c|c|c|c|c|}
\hline & $\begin{array}{l}\text { Outpatient } \\
\text { cost per visit }\end{array}$ & $\begin{array}{l}\text { Hospitalization } \\
\text { cost per visit }\end{array}$ & DMC & DNMC & IC & Total cost \\
\hline \multicolumn{7}{|l|}{ City } \\
\hline \multirow[t]{2}{*}{ Beijing } & $434(598 \pm 901)$ & $0(6,120 \pm \mid 7,755)$ & $6,824(15,519 \pm 28,396)$ & $30(680 \pm I, 378)$ & $0(5,737 \pm 12,532)$ & II,038 $(22,199 \pm 35,174)$ \\
\hline & $(67[93 \pm 139])$ & (0 [947 $\pm 2,749])$ & $(1,057[2,403 \pm 4,396])$ & $(5[105 \pm 2 \mid 3])$ & $(0[888 \pm 1,940])$ & $(I, 709[3,437 \pm 5,446])$ \\
\hline \multirow[t]{2}{*}{ Guangzhou } & $190(278 \pm 377)$ & $0(3,938 \pm 8,3 \mid 8)$ & $3, I 78(\mid 2,45 I \pm 27,50 I)$ & $0(308 \pm I, 062)$ & $0(558 \pm 3,135)$ & $3,773(13,277 \pm 28,759)$ \\
\hline & $(29[43 \pm 58])$ & $(0[6 \mid 0 \pm 1,288])$ & $(492[1,928 \pm 4,258])$ & $(0[48 \pm 164])$ & $(0[86 \pm 485])$ & $(584[2,056 \pm 4,453])$ \\
\hline \multirow[t]{2}{*}{ Shanghai } & $212(298 \pm 300)$ & $0(3,|7| \pm 5,644)$ & $5,214(10,236 \pm 14,628)$ & $0(848 \pm 3,691)$ & $0(1,456 \pm 8,436)$ & $6,473(12,676 \pm \mid 7,623)$ \\
\hline & $(33[46 \pm 46])$ & $(0[49 \mid \pm 874])$ & $(807[1,585 \pm 2,265])$ & $(0[13| \pm 57|])$ & $(0[225 \pm I, 306])$ & $(1,002[1,963 \pm 2,728])$ \\
\hline \multirow[t]{2}{*}{ Chengdu } & $0(97 \pm 343)$ & $3,695(5,00 I \pm 7,824)$ & $6,628(9,57| \pm| 4,376)$ & $0(334 \pm I, 0 I I)$ & $0(6 \mid 4 \pm 3,058)$ & $7,200(10,510 \pm 14,934)$ \\
\hline & $(0[15 \pm 53])$ & $(572[774 \pm I, 2 \mid I])$ & $(1,026[1,482 \pm 2,226])$ & $(0[52 \pm 157])$ & $(0[95 \pm 473])$ & $(1, I I 5[1,627 \pm 2,3 \mid 2])$ \\
\hline$P$-value & $<0.001$ & $<0.001$ & 0.012 & $<0.001$ & $<0.001$ & $<0.001$ \\
\hline \multicolumn{7}{|l|}{ Sex } \\
\hline \multirow[t]{2}{*}{ Male } & $156(304 \pm 556)$ & $0(4,494 \pm$ I I,635) & $5,977(11,963 \pm 23,020)$ & $0(534 \pm 2,330)$ & $0(1,529 \pm 6,944)$ & $6,397(|4| 23 \pm 25,,05 I)$ \\
\hline & $(24[47 \pm 86])$ & $(0[696 \pm 1,801])$ & $(925[1,852 \pm 3,564])$ & $(0[83 \pm 36 I])$ & $(0[237 \pm I, 075])$ & $(990[2,187 \pm 3,879])$ \\
\hline \multirow[t]{2}{*}{ Female } & $164(360 \pm 596)$ & $0(4,7 \mid 5 \pm 8,980)$ & $4,712(11,982 \pm 20,804)$ & $8(554 \pm I, 257)$ & $0(3,583 \pm 10,505)$ & $6,413(16,223 \pm 28,199)$ \\
\hline & $(25[56 \pm 92])$ & $(0[730 \pm I, 390])$ & $(730[1,855 \pm 3,221])$ & $(1[86 \pm 195])$ & $(0[555 \pm 1,627])$ & $(993[2,512 \pm 4,366])$ \\
\hline$P$-value & 0.67 & 0.704 & 0.71 & 0.004 & $<\mathbf{0 . 0 0 1}$ & 0.726 \\
\hline \multicolumn{7}{|l|}{ Age (years) } \\
\hline \multirow[t]{2}{*}{$\leq 60$} & $257(35 I \pm 405)$ & $0(1,429 \pm 3,483)$ & $3736(5592 \pm 6583)$ & $0(121 \pm 269)$ & $0(3,09| \pm I I| 89)$, & $3,850(8,804 \pm \mid 4,253)$ \\
\hline & $(40[54 \pm 63])$ & $(0[22 I \pm 539])$ & $(578[866 \pm 1,019])$ & $(0[19 \pm 42])$ & $(0[479 \pm 1,732])$ & $(596[1,363 \pm 2,207])$ \\
\hline \multirow[t]{2}{*}{$>60$} & $144(3 \mid 4 \pm 598)$ & $0(5,165 \pm 11,806)$ & $6,096(13,083 \pm 23,564)$ & $0(626 \pm 2,296)$ & $0(1,726 \pm 6,194)$ & $7,07 \mid(\mid 5,580 \pm 25,846)$ \\
\hline & $(22[49 \pm 93])$ & $(0[800 \pm I, 828])$ & $(944[2,026 \pm 3,648])$ & $(0[97 \pm 356])$ & $(0[267 \pm 959])$ & $(1,095[2,4 \mid 2 \pm 4,002])$ \\
\hline$P$-value & 0.006 & $<0.001$ & 0.003 & 0.094 & 0.204 & 0.006 \\
\hline \multicolumn{7}{|c|}{ Household income per capita monthly, RMB Yuan (US\$) } \\
\hline \multirow[t]{2}{*}{$<\mathrm{I}, 000(\mathrm{I} 54)$} & $100(280 \pm 430)$ & $0(5,562 \pm 9,291)$ & $7,128(13,482 \pm 19,810)$ & $6(357 \pm 959)$ & $0(3,278 \pm 9,036)$ & $7,533(17,368 \pm 26,818)$ \\
\hline & $(15[43 \pm 67])$ & $(0[86 I \pm I, 439])$ & $(1,104[2,087 \pm 3,067])$ & $(I[55 \pm \mid 49])$ & $(0[508 \pm I, 399])$ & $(1,166[2,689 \pm 4,152])$ \\
\hline $1,000-1,999$ & $46(24 I \pm 455)$ & $2862(4,35 I \pm 5,394)$ & $6,993(10,816 \pm 14,315)$ & $0(404 \pm 990)$ & $0(2,009 \pm 8,801)$ & $7,666(13,220 \pm \mid 7,873)$ \\
\hline$(154-308)$ & $(7[37 \pm 70])$ & $(443[674 \pm 835])$ & $(1083[1,675 \pm 2,216])$ & $(0[63 \pm 153])$ & $(0[3 \mid I \pm I, 363])$ & $(I, 187[2,047 \pm 2,767])$ \\
\hline $2,000-2,999$ & $179(363 \pm 733)$ & $0(4,120 \pm 7,560)$ & $5,429(12,676 \pm 23,824)$ & $0(704 \pm 3,081)$ & $0(2,446 \pm 9,346)$ & $6,825(15,953 \pm 28,895)$ \\
\hline$(309-463)$ & $(28[56 \pm I \mid 4])$ & $(0[638 \pm I,|7|])$ & $(84 \mid[1,963 \pm 3,689])$ & $(0[109 \pm 477])$ & $(0[379 \pm \mathrm{I}, 447])$ & $(1,057[2,470 \pm 4,474])$ \\
\hline $3,000-3,999$ & $198(34 I \pm 484)$ & $0(4,522 \pm 10,1 \mathrm{II})$ & $4,532(12,038 \pm 28,767)$ & $0(487 \pm I, 442)$ & $0(2,539 \pm 7,358)$ & $5,4 \mid 2(15,173 \pm 30,497)$ \\
\hline$(464-618)$ & $(31[53 \pm 75])$ & $(0[700 \pm 1565])$ & $(702[1,864 \pm 4,454])$ & $(0[75 \pm 223])$ & $(0[393 \pm I, 139])$ & $(838[2,349 \pm 4,722])$ \\
\hline \multirow[t]{2}{*}{$\geq 4,000$ (619) } & $232(372 \pm 503)$ & $0(5,076 \pm 19,386)$ & $3,348(11,455 \pm 23,636)$ & $0(594 \pm 2,053)$ & $0(I, 04 I \pm 4,493)$ & $3,756(\mid 3,276 \pm 25,90 I)$ \\
\hline & $(36[58 \pm 78])$ & $(0[786 \pm 3,001])$ & $(518[1,774 \pm 3,660])$ & $(0[92 \pm 3 \mid 8])$ & $(0[161 \pm 696])$ & $(582[2,056 \pm 4,0 \mid 0])$ \\
\hline$P$-value & $<0.001$ & 0.003 & 0.54 & 0.549 & 0.464 & 0.632 \\
\hline \multicolumn{7}{|c|}{ Having medical insurance } \\
\hline \multirow[t]{2}{*}{ Yes } & $157(322 \pm 575)$ & $0(4,576 \pm I I, 097)$ & $5,750(12,1 \mid 12 \pm 22,706)$ & $0(553 \pm 2,133)$ & $0(2,105 \pm 8,234)$ & $6,534(14,889 \pm 26,309)$ \\
\hline & $(24[50 \pm 89])$ & $(0[709 \pm I, 7 \mid 8])$ & $(890[1,875 \pm 3,5 \mid 5])$ & $(0[86 \pm 330])$ & $(0[326 \pm 1,275])$ & $(1,012[2,305 \pm 4,073])$ \\
\hline \multirow[t]{2}{*}{ No } & $148(263 \pm 360)$ & $0(4,062 \pm 7,947)$ & $2,954(8758 \pm 14,656)$ & $0(247 \pm 754)$ & $0(1,688 \pm 4,857)$ & $2,954(10,447 \pm 15,603)$ \\
\hline & $(23[4 I \pm 56])$ & $(0[629 \pm 1,230])$ & $(457[1,356 \pm 2,269])$ & $(0[38 \pm 117])$ & $(0[26 I \pm 752])$ & $(457[1,6|8 \pm 2,4| 6])$ \\
\hline$P$-value & 0.535 & 0.445 & 0.176 & 0.14 & 0.953 & 0.183 \\
\hline \multicolumn{7}{|c|}{ Current severity of COPD } \\
\hline Mild & $122(|95 \pm 28|)$ & $0(I, 908 \pm 5,40 I)$ & $2,288(5,|4| \pm \mid 2,953)$ & $0(589 \pm 2,7 \mid 8)$ & $0(1,107 \pm 4,076)$ & $2,857(6,837 \pm|4,7| 0)$ \\
\hline & $(19[30 \pm 43])$ & $(0[295 \pm 836])$ & $(354[796 \pm 2,006])$ & $(0[9 I \pm 42 \mid])$ & $(0[|7| \pm 63 \mid])$ & $(442[1,059 \pm 2,278])$ \\
\hline Moderate & $|7|(325 \pm 487)$ & $0(4,463 \pm 14,380)$ & $4,257(10,280 \pm 19,986)$ & $0(525 \pm 2,329)$ & $0(2,629 \pm 9,055)$ & $4,870(13,553 \pm 26,173)$ \\
\hline & $(26[50 \pm 75])$ & $(0[691 \pm 2226])$ & $(659[1,592 \pm 3,094])$ & $(0[8 I \pm 36 \mid])$ & $(0[407 \pm \mathrm{I}, 402])$ & $(754[2,098 \pm 4,052])$ \\
\hline Severe & $161(339 \pm 493)$ & $0(5,7|2 \pm 7,8| 4)$ & $8,408(15,802 \pm 25,838)$ & $10(527 \pm 1,233)$ & $0(2,346 \pm 9,844)$ & $10,350(18,875 \pm 28,178)$ \\
\hline & $(25[52 \pm 76])$ & $(0[884 \pm I, 2 \mid 0])$ & $(1,302[2,447 \pm 4,000])$ & $(2[82 \pm 191])$ & $(0[363 \pm I, 524])$ & $(1,602[2,922 \pm 4,363])$ \\
\hline Very severe & $227(5 \mid 8 \pm 1215)$ & $6,516(7,628 \pm 7,816)$ & $I, I 3 I 6(2,494 I \pm 3 I, 692)$ & $0(531 \pm 998)$ & $0(|, 0| 4 \pm 2,9 \mid 3)$ & $12,359(26,377 \pm 32,059)$ \\
\hline & $(35[80 \pm \mid 88])$ & $(1,009[1,|8| \pm 1,2 \mid 0])$ & $(1,752[3,862 \pm 4,907])$ & $(0[82 \pm 154])$ & $(0[157 \pm 45 \mid])$ & $(1,913[4,084 \pm 4,964])$ \\
\hline$P$-value & 0.026 & $<0.001$ & $<0.001$ & 0.002 & 0.75 & $<0.001$ \\
\hline Number of con & bidities & & & & & \\
\hline 0 & $184(309 \pm 418)$ & $0(3,55| \pm| 8,202)$ & $2,977(7,693 \pm 19,428)$ & $0(548 \pm 3,577)$ & $0(I, 3 \mid I \pm 8,768)$ & $3,048(9,503 \pm 22,543)$ \\
\hline & $(28[48 \pm 65])$ & $(0[550 \pm 2,8 \mid 8])$ & $(46 \mid[|| 9 \mid \pm 3,008])$, & $(0[85 \pm 554])$ & $(0[203 \pm 1,357])$ & $(472[I, 47 I \pm 3,490])$ \\
\hline 1 & $146(205 \pm 272)$ & $0(3,986 \pm 6,470)$ & $4,874(10,138 \pm 17,466)$ & $0(397 \pm I, 582)$ & $0(9 \mid 8 \pm 4,305)$ & $5,412(11,487 \pm 19,427)$ \\
\hline & $(23[32 \pm 42])$ & $(0[6 \mid 7 \pm I, 002])$ & $(755[1,570 \pm 2,704])$ & $(0[62 \pm 245])$ & $(0[142 \pm 666])$ & $(838[1,779 \pm 3,008])$ \\
\hline
\end{tabular}

(Continued) 
Table 3 (Continued)

\begin{tabular}{|c|c|c|c|c|c|c|}
\hline & $\begin{array}{l}\text { Outpatient } \\
\text { cost per visit }\end{array}$ & $\begin{array}{l}\text { Hospitalization } \\
\text { cost per visit }\end{array}$ & DMC & DNMC & IC & Total cost \\
\hline \multirow[t]{2}{*}{2} & $106(362 \pm 837)$ & $0(4,992 \pm 9,45 I)$ & $6,198(13,5 \mid 3 \pm 23,177)$ & $0(526 \pm I, 278)$ & $0(1,695 \pm 4,815)$ & $7,679(16,014 \pm 24,356)$ \\
\hline & $(16[56 \pm 130])$ & $(0[773 \pm I, 463])$ & $(960[2,092 \pm 3,589])$ & $(0[8| \pm| 98])$ & $(0[262 \pm 745])$ & $(I, 189[2,479 \pm 3,77 \mid])$ \\
\hline \multirow[t]{2}{*}{$\geq 3$} & $255(476 \pm 625)$ & $2,765(6,103 \pm 8,47 I)$ & $9,222(17,803 \pm 29,847)$ & $40(803 \pm I, 477)$ & $0(5,506 \pm 13,383)$ & $13,192(24,263 \pm 36,730)$ \\
\hline & $(39[74 \pm 97])$ & $(428[945 \pm I, 312])$ & $(I, 428[2,756 \pm 4,62 I])$ & $(6[124 \pm 229])$ & $(0[852 \pm 2,072])$ & $(2,043[3,757 \pm 5,687])$ \\
\hline$P$-value & 0.002 & $<0.001$ & $<0.001$ & $<\mathbf{0 . 0 0 1}$ & $<\mathbf{0 . 0 0 1}$ & $<0.001$ \\
\hline
\end{tabular}

Notes: Data were presented as median (mean \pm standard deviation). Kruskal-Wallis or Wilcoxon test was to test the differences of costs among groups. Values in bold indicated statistical significance.

Abbreviations: COPD, chronic obstructive pulmonary disease; DMC, direct medical cost; DNMC, direct nonmedical cost; IC, indirect cost.

which were lower than those reported in previous studies conducted in 2006 and 2008-2009, ${ }^{7,15}$ but the annual direct expenditure was much higher than that estimated in the US (only $12.1 \%$, US\$ 6,030/49,800). ${ }^{16}$ The annual DMCs were higher than those reported in 2006 in China, ${ }^{7}$ which were likely due to larger aging population and more expensive health care costs. A lower proportion of direct costs to personal income could have resulted from the rapid socioeconomic development recently in China. ICs accounted for $\sim 14 \%$ of total costs, which was lower than that reported in the US $(27 \%-61 \%) .{ }^{17}$ One study noted that there was an increase in ICs on COPD in the past several years. ${ }^{18}$

Table 4 Factors related to total costs due to COPD

\begin{tabular}{|c|c|c|c|c|c|c|}
\hline Factors & Low burden, $\mathbf{n}$ & High burden, $\mathbf{n}$ & cOR $(95 \% \mathrm{Cl})$ & $P$-value & aOR $(95 \% \mathrm{Cl})^{\mathrm{a}}$ & $P$-value \\
\hline \multicolumn{7}{|l|}{ Sex } \\
\hline Female & 128 & 53 & I & & I & \\
\hline Male & 370 & 113 & $0.74(0.50,1.08)$ & 0.12 & $0.76(0.46,1.25)$ & 0.284 \\
\hline \multicolumn{7}{|l|}{ Age (years) } \\
\hline$\leq 60$ & 102 & 13 & I & & I & \\
\hline$>60$ & 391 & 152 & $3.05(1.66,5.60)$ & $<\mathbf{0 . 0 0 1}$ & $2.36(1.18,4.72)$ & 0.015 \\
\hline \multicolumn{7}{|l|}{ Education (years) } \\
\hline$\leq 9$ & 290 & 114 & $1.94(1.09,3.46)$ & 0.025 & $1.45(0.72,2.93)$ & 0.294 \\
\hline $10-12$ & 128 & 36 & $1.39(0.72,2.67)$ & 0.324 & $1.46(0.68,3.15)$ & 0.334 \\
\hline$>12$ & 79 & 16 & I & & I & \\
\hline Duration of disease in years & $3.00(8.72 \pm \mathrm{I} I .86)^{\mathrm{b}}$ & $5.00(9.74 \pm \mathrm{II} .12)^{\mathrm{b}}$ & $1.01(0.99,1.02)$ & 0.334 & $0.99(0.97,1.00)$ & 0.136 \\
\hline \multicolumn{7}{|l|}{ Having medical insurance } \\
\hline Yes & 476 & 159 & I $(0.42,2.40)$ & 0.996 & $0.60(0.20,1.86)$ & 0.378 \\
\hline No & 21 & 7 & 1 & & I & \\
\hline \multicolumn{7}{|l|}{ Number of comorbidities } \\
\hline 0 & 122 & 18 & I & & I & \\
\hline I & 184 & 43 & $1.58(0.87,2.87)$ & 0.13 & $1.06(0.51,2.19)$ & 0.873 \\
\hline 2 & 115 & 51 & $3.01(1.66,5.45)$ & $<0.001$ & $1.87(0.88,3.96)$ & 0.104 \\
\hline$\geq 3$ & 76 & 54 & $4.82(2.63,8.82)$ & $<\mathbf{0 . 0 0 1}$ & $2.05(0.93,4.54)$ & 0.076 \\
\hline \multicolumn{7}{|c|}{ Monthly family per capita income, RMB Yuan (US\$) } \\
\hline$<1,000(154)$ & 26 & 12 & I & & I & \\
\hline I,000-I,999 (I54-308) & 144 & 50 & $0.75(0.35,1.60)$ & $0.46 I$ & $0.72(0.28,1.88)$ & 0.503 \\
\hline $2,000-2,999(309-463)$ & 154 & 51 & $0.72(0.34,1.53)$ & 0.388 & $0.76(0.29,2.01)$ & 0.586 \\
\hline $3,000-3,999(464-618)$ & 69 & 24 & $0.75(0.33,1.72)$ & 0.503 & $0.79(0.28,2.24)$ & 0.660 \\
\hline$\geq 4,000$ (619) & 101 & 28 & $0.6(0.27,1.34)$ & 0.213 & $0.66(0.23,1.86)$ & 0.427 \\
\hline \multicolumn{7}{|l|}{ Current disease severity } \\
\hline Mild & 126 & 12 & I & & I & \\
\hline Moderate & 219 & 64 & $3.07(1.60,5.90)$ & 0.001 & $3.26(1.55,6.87)$ & 0.002 \\
\hline Severe & 110 & 64 & $6.11(3.13,11.91)$ & $<\mathbf{0 . 0 0 1}$ & $4.17(1.91,9.09)$ & $<\mathbf{0 . 0 0 1}$ \\
\hline Very severe & 35 & 24 & $7.2(3.28,15.83)$ & $<0.001$ & $4.43(1.76,11.13)$ & 0.002 \\
\hline \multicolumn{7}{|l|}{ Exacerbations } \\
\hline No & 437 & 47 & I & & I & \\
\hline Yes & 94 & 68 & $6.73(4.36,10.38)$ & $<0.001$ & $4.68(2.71,8.08)$ & $<0.001$ \\
\hline Number of exacerbations & $1.00(1.17 \pm 1.76)$ & $2.00(2.4 \mathrm{I} \pm 2.0 \mathrm{I})$ & $1.38(1.25,1.52)$ & $<\mathbf{0 . 0 0 1}$ & $1.27(1.13,1.43)$ & $<\mathbf{0 . 0 0 1}$ \\
\hline
\end{tabular}

Notes: ${ }^{a}$ Adjusted for city and other variables. ${ }^{b}$ Data shown as median (mean \pm standard deviation). Values in bold indicated significance, $P<0.05$.

Abbreviations: $\mathrm{Cl}$, confidence interval; COPD, chronic obstructive pulmonary disease; cOR, crude odds ratio; aOR, adjusted odds ratio. 
In this study, hospitalization costs accounted for $56.7 \%$ of the total costs, which were consistent with some prior studies. ${ }^{7,19-21}$ However, one study conducted in rural areas of China found that the direct costs were mainly driven by the costs of outpatient services (42.3\%), followed by hospitalization costs $(32.5 \%) .{ }^{15}$ The difference between urban and rural areas may be due to health care disparities and different distributions of severity.

This study also revealed that age, disease severity, and acute exacerbations were the independent factors for high economic burden in COPD patients. In urban areas of China, COPD patients who were older and had more severe condition and higher number of acute exacerbations had a greater risk of high economic burden. The elderly generally have a high need for hospitalizations and caregivers partly due to increased risks of comorbidities and poor physical conditions. ${ }^{17}$ Previous studies had shown the relationship between costs of COPD and acute exacerbation, ${ }^{9,22,23}$ which was consistent with the results of this study. Acute exacerbation was a major cause of hospitalization. ${ }^{23,24}$ Also, exacerbations became more frequent and more severe with the severity of COPD ${ }^{25}$ And as mentioned earlier, hospitalization was identified as the largest cost category, and therefore, some measures, such as pulmonary rehabilitation, outreach nursing, multidisciplinary care, patient self-management, patient counseling and education, case management, and outreach follow-up, or services provided after discharge to decrease hospitalizations for COPD exacerbations could be helpful to cut the costs on COPD. ${ }^{26-29}$

This was a multi-center cross-sectional study conducted in urban areas of China. There were some strengths and limitations. This study was carried out in four metropolitan areas with a high response rate of $98 \%$. The study population might well represent Chinese metropolitan population, but caution should be taken when generalizing the findings to other populations. The national economic burden estimate may be biases due to the difference in costs between rural and urban areas. As a cross-sectional study, there is a limitation for assuming causal associations between the influencing factors and costs of COPD. Another limitation was that the COPD patients of the study were not randomly selected but were selected from only four metropolitan areas of the country, which may affect the external validity. A convenient sample might be subject to selection bias although no serious ones had been identified for the study. In addition, recall biases might exist although some self-reporting information was confirmed by reviewing medical charts and searching medical records.

\section{Conclusion}

COPD posed a heavy economic burden in China. Hospitalization was a major driver of the costs related to COPD. Aging, COPD severity, and acute exacerbations increased the risk of high costs for COPD.

\section{Acknowledgments}

This study was sponsored by Novartis (China) Investment Co. Ltd and supported by Shanghai Leading Academic Discipline Project of Public Health (Project Number: 15GWZK80101). The authors would like to thank Professor Chen Weiqing, Professor Luan Rongsheng, and Doctor Liu Zhaolan for helping during data collection.

\section{Disclosure}

The authors report no conflicts of interest in this work.

\section{References}

1. Fang $X$, Wang $X$, Bai C. COPD in China: the burden and importance of proper management. Chest. 2011;139(4):920-929.

2. World Health Organization. Chronic Obstructive Pulmonary Disease (COPD). Available from: http://www.who.int/mediacentre/factsheets/ fs 315/en/. Accessed October 6, 2016.

3. Zhong N, Wang C, Yao W, et al. Prevalence of chronic obstructive pulmonary disease in China: a large, population-based survey. Am J Respir Crit Care Med. 2007;176(8):753-760.

4. Yang G, Wang Y, Zeng Y, et al. Rapid health transition in China, 1990-2010: findings from the Global Burden of Disease Study 2010. Lancet. 2013;381(9882):1987-2015.

5. Guarascio AJ, Ray SM, Finch CK, Self TH. The clinical and economic burden of chronic obstructive pulmonary disease in the USA Clinicoecon Outcomes Res. 2013;5:235-245.

6. Dang-Tan T, Ismaila A, Zhang S, et al. Clinical, humanistic, and economic burden of chronic obstructive pulmonary (COPD) in Canada: a systematic review. BMC Res Notes. 2015;8:464.

7. He QY, Zhou X, Xie CM, et al. Impact of chronic obstructive pulmonary disease on quality of life and economic burden in Chinese urban areas. Zhonghua Jie He He Hu Xi Za Zhi. 2009;32(4):253-257.

8. Zhang B, Zhang Y, Yang J, et al. Analysis on direct economic burden of community COPD patients and its influence factors in Chengdu. Wei Sheng Yan Jiu. 2007;36(6):706-710.

9. Chen YH, Yao WZ, Cai BQ, et al. Economic analysis in admitted patients with acute exacerbation of chronic obstructive pulmonary disease. Chin Med J (Engl). 2008;121(7):587-591.

10. Dalal AA, Christensen L, Liu F, Riedel AA. Direct costs of chronic obstructive pulmonary disease among managed care patients. Int $J$ Chron Obstruct Pulmon Dis. 2010;5:341-349.

11. Chapman KR, Bourbeau J, Rance L. The burden of COPD in Canada: results from the Confronting COPD survey. Respir Med.2003;97(supplC): S23-S31.

12. Wu M, Zhao Q, Chen Y, Fu C, Xu B. Quality of life and its association with direct medical costs for COPD in urban China. Health Qual Life Outcomes. 2015;13:57.

13. National Bureau of Statistics of China [webpage on the Internet]. China Statistical Yearbook. Available from: http:/www.stats.gov.cn/tjsj/ ndsj/2011/indexch.htm. Accessed March 10, 2015.

14. National Health and Family Planning Commission of the People's Republic of China. China Health Statistical Yearbook. Available from: http://www.moh.gov.cn/zwgkzt/ptjty/201206/55044/files/3ca7756121 334b7a870a25ac79988f23.pdf. Accessed March 10, 2015. 
15. Lou P, Zhu Y, Chen P, et al. Vulnerability, beliefs, treatments and economic burden of chronic obstructive pulmonary disease in rural areas in China: a cross-sectional study. BMC Public Health. 2012;12:287.

16. Ford ES, Murphy LB, Khavjou O, Giles WH, Holt JB, Croft JB. Total and state-specific medical and absenteeism costs of COPD among adults aged $\geq 18$ years in the United States for 2010 and projections through 2020. Chest. 2015;147(1):31-45.

17. Patel JG, Nagar SP, Dalal AA. Indirect costs in chronic obstructive pulmonary disease: a review of the economic burden on employers and individuals in the United States. Int J Chron Obstruct Pulmon Dis. 2014;9:289-300.

18. Herse F, Kiljander T, Lehtimaki L. Annual costs of chronic obstructive pulmonary disease in Finland during 1996-2006 and a prediction model for 2007-2030. NPJ Prim Care Respir Med. 2015;25:15015.

19. Tan WC, Ng TP. COPD in Asia: where East meets West. Chest. 2008; 133(2):517-527.

20. Miravitlles M, García-Polo C, Domenech A, Villegas G, Conget F, de la Roza C. Clinical outcomes and cost analysis of exacerbations in chronic obstructive pulmonary disease. Lung. 2013;191(5):523-530.

21. Wilson L, Devine EB, So K. Direct medical costs of chronic obstructive pulmonary disease: chronic bronchitis and emphysema. Respir Med. 2000;94(3):204-213.

22. Toy EL, Gallagher KF, Stanley EL, Swensen AR, Duh MS. The economic impact of exacerbations of chronic obstructive pulmonary disease and exacerbation definition: a review. COPD. 2010;7(3):214-228.
23. Perera PN, Armstrong EP, Sherrill DL, Skrepnek GH. Acute exacerbations of COPD in the United States: inpatient burden and predictors of costs and mortality. COPD. 2012;9(2):131-141.

24. Ozkaya S, Findik S, Atici AG. The costs of hospitalization in patients with acute exacerbation of chronic obstructive pulmonary disease. Clinicoecon Outcomes Res. 2011;3:15-18.

25. Hurst JR, Vestbo J, Anzueto A, et al. Susceptibility to exacerbation in chronic obstructive pulmonary disease. N Engl J Med. 2010;363(12): 1128-1138.

26. Casas A, Troosters T, Garcia-Aymerich J, et al. Integrated care prevents hospitalizations for exacerbations in COPD patients. Eur Respir J. 2006; 28(1):123-130.

27. Bourbeau J, Julien M, Maltais F, et al. Reduction of hospital utilization in patients with chronic obstructive pulmonary disease: a disease specific self-management intervention. Arch Intern Med. 2003;163(5): 585-591.

28. Peytremann-Bridevaux I, Staeger P, Bridevaux PO, Ghali WA, Burnand B. Effectiveness of chronic obstructive pulmonary diseasemanagement programs: systematic review and meta-analysis. Am J Med. 2008;121(5):433-443.

29. Denberg TD, Lin CT, Myers BA, Cashman JM, Kutner JS, Steiner JF. Improving patient care through health-promotion outreach. J Ambul Care Manage. 2008;31(1):76-87.
International Journal of COPD

\section{Publish your work in this journal}

The International Journal of COPD is an international, peer-reviewed journal of therapeutics and pharmacology focusing on concise rapid reporting of clinical studies and reviews in COPD. Special focus is given to the pathophysiological processes underlying the disease, intervention programs, patient focused education, and self management protocols.

\section{Dovepress}

This journal is indexed on PubMed Central, MedLine and CAS. The manuscript management system is completely online and includes a very quick and fair peer-review system, which is all easy to use. Visit http://www.dovepress.com/testimonials.php to read real quotes from published authors. 